\title{
An Adaptive Radio Link Protocol to Improve TCP Performance over Correlated Fading Wireless Channels
}

\author{
Haitao Lin ${ }^{1}$, Sajal K. Das ${ }^{1}$, and Hee Yong Youn ${ }^{2}$ \\ 1 Center for Research in Wireless Mobility and Networking (CReWMaN) \\ Department of Computer Science and Engineering \\ University of Texas at Arlington \\ Arlington, TX 76019, USA \\ Telephone:+1 817-272-7409 \\ Fax:+1 817-272-3784 \\ $\{$ hlin, das\}@cse.uta.edu \\ http://crewman.uta.edu \\ 2 School of Information and Communication Engineering \\ Sungkyunkwan University \\ Jangangu Chunchundong 300 \\ Suwon, Korea, 440-746 \\ Telephone:+82 31-290-7147 \\ Fax:+82 31-290-7231 \\ youn@ece.skku.ac.kr \\ http://csn.skku.ac.kr
}

\begin{abstract}
Improving Internet service performance is essential for the future wireless networks. The correlated fading channels are found very unfriendly to TCP performance in such networks. Moreover, the variation in fading rate brings further challenge to wireless networks when supporting Internet applications, most of which runs TCP as the transport layer protocol. In this paper, we first analytically study the performance of different RLP (Radio Link Protocol) retransmission schemes in supporting TCP. An analytic metric called RLP retransmission efficiency is introduced to evaluate performance of different RLP retransmission schemes under correlated fading channels. With analytic and simulation results, we show that a single retransmission scheme does not yield the best performance over different rates of fading. Based on this observation, we propose an Adaptive Radio Link Protocol (ARLP) that has a fading rate detector to estimate the channel fading rate. Based on the estimated channel fading rate, ARLP adaptively adjusts its retransmission schemes to achieve the optimal data transmission performance. Simulation results show that ARLP can improve both the performance of a particular mobile host and the total throughput of wireless networks.
\end{abstract}




\section{Introduction}

TCP performance over wireless channels has been studied extensively in recent years. The major effort is focused on avoiding TCP's congestion control mechanism from being initiated when packet losses happen over unreliable wireless links. Proposals addressing this problem can be divided into three classes: i) Splitting the TCP connection into a wireline segment and a wireless segment [1] 2]; ii) designing new TCPs such as W-TCP [11] or modifying normal TCP as to differentiate between losses over wireless links and real congestions; iii) Using local retransmissions in link layer or MAC layer so that most packet losses are recovered before TCP times out. In this way, TCP is presented with a much more reliable link. The unnecessary initiations of TCP's congestion control mechanism are avoided and throughput is improved. Due to the inherent problems in the first and second classes of mechanisms to address this problem, only local retransmission solutions are commercially implemented. In cdma2000 (IS2000) systems, a link layer protocol called Radio Link Protocol (RLP) is introduced to recover data losses over wireless links [14. Similar protocol called RLC (Radio Link Control) is utilized in UMTS system [12]. The commercial implementations of local retransmission schemes imply their important roles in solving TCP over wireless problem, and any improvement to their performance is very important to the future wireless networks.

On the other hand, the local retransmission is a particular implementation of Automatic Repeat reQuest (ARQ). For example, the RLP used in cdma2000 is a negative acknowledgement (NACK) based Selective-Repeat ARQ, with finite number of retransmissions. It has been proposed by many researchers that the link layer protocol over wireless channels should be adaptive to the varying channel characteristics as wireless channels experience time-varying fading most of the time. Many adaptive ARQ schemes have been proposed [3 [4] [7] In [3], three adaptive hybrid error control schemes are proposed and compared. These schemes differ in two aspects: how many Reed-Solomon (RS) code segments are used for each packet and how a packet with uncorrectable errors are retransmitted. Hlaing [4] proposed to use XOR-ing to do bit error rate (BER) estimation, and to use Frequency Hoping to counteract long deep fading. Paul and Mani [7] proposed to use adaptive MAC frame length to improve the link layer protocol performance over time-varying fading channels. In general, among all these works, adaptiveness is incorporated into link layer protocol in several ways. First, the coding rate can be adaptive: the general idea is that for high bit-error-rate (BER) channels, use more robust error correction coding, which results in lower effective data rates; for low BER channels, use less robust coding, with higher effective data rate. Second, the use of ARQ can be adaptive: for low BER channels, use Forward Error Correction (FEC) coding to correct the errors, which yield higher throughput in this environment; for high BER channels, use ARQ to recover the erroneous data since ARQ is more efficient in this situation. Third, the size of the atomic transmission unit can be adaptive: it is desirable to use larger frame size over low BER channels; while using smaller frame size over high BER channels yields better performance. In this paper, we explore another possibility of adaptiveness: the way to do retransmissions in 
ARQ. By introducing this adaptiveness, we solve the problem of how to optimize the ARQ retransmission efficiency in a time-varying correlated fading channel, especially when the rate of fading varies. This problem is extremely important as it is closely related to the problem of how to further improve the link layer performance in supporting TCP. As mentioned, the local retransmission schemes like RLP are nothing but particular implementations or variants of the standard ARQs.

In the RLP specified in cdma2000 [14], when an RLP frame is found in error and can not be recovered by FEC, the frame is retransmitted for a finite number of times, and each time the number of copies of the retransmitted frame can be different. For example, for the first time of retransmission, two copies of the lost frame can be sent in two consecutive time slots. If both of these copies do not get through and time out happens, another 3 copies of this frame are transmitted. If all of these retransmissions fail, RLP gives up and let upper layers like TCP recover the lost data. This particular way of doing retransmission is called the RLP scheme $(1,2,3)$, which involves first a one-copy transmission, then a twocopy retransmission, followed by a three-copy retransmission. There are several other retransmission schemes, such as $(1,1,1,1,1,1)$ and $(1,1,2,3)$, etc. For detailed information on RLP, refer to [14.

In this paper, we first show that over correlated fading channels of different characteristics, RLP retransmission schemes perform differently and have different retransmission efficiency. More specifically, scheme $(1,2,3)$ performs better over fast fading channels, where the channel states of two consecutive frames have low correlation; while scheme $(1,1,1,1,1,1)$ performs better over slow fading channels, where the channel states of two consecutive frames have high correlation. We present both analytical metric and experimental results to support this finding because of its significance: it serves as the primary motivation and the basic supporting fact of our proposed Adaptive Radio Link Protocol (ARLP).

ARLP enhances the link layer protocol performance over correlated fading channels where fading rate varies. The protocol consists of a simple but efficient sequential test algorithm to determine the fading rate of the channel, and a modified RLP receiver to respond to the detected fading rate changes with corresponding retransmission scheme that performs best with the detected fading rate. As a result, the ARQ retransmission efficiency is optimized.

The rest of this paper is organized as follows: Section 2 discusses the performance of two typical RLP retransmission schemes, their difference and implications. Section 3 presents the proposed adaptive RLP, including sequential test algorithm and the adaptive retransmission algorithm. Section 4 evaluates the performance of the proposed protocol ARLP and compares it with the standard RLP. Section 5 concludes the paper.

\section{RLP Performance Study}

We start by briefly introducing the wireless correlated fading channel model. The two-state first order Markov chain has been widely adopted as a mathematical tool to model the wireless fading channels because of its accuracy and its ease 


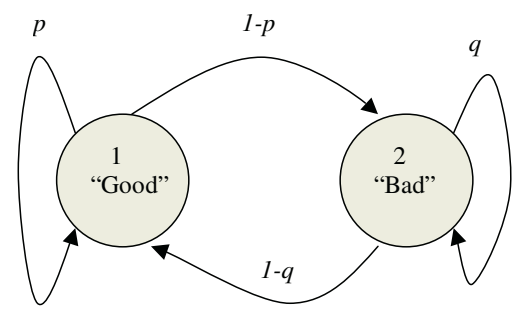

Fig. 1. Two-state Markov Chain

to mathematically adopt into analysis. When the wireless channel is subject to correlated multipath fading, the probabilities of getting an error among the bits being transmitted are not independent and identically distributed (i.i.d.) 8]. Instead, there are high correlations between consecutive bits being transmitted. If we assume the channel state does not change during the transmission of a bit, which is true for high speed data transmission or slow moving speed, the wireless channel can be modeled as a two-state Markov chain 99 [10]. If we further assume that the channel state during transmission of a link layer frame does not change, the two-state Markov chain can be applied to the frame level channel error modeling and has been shown to be an accurate model [10. In this section, we investigate the system performance based on this channel model.

\subsection{Correlated Fading Channel Model}

The frame error model can be approximated by a simple two-state Markov chain [10] as shown in Fig. 1] Assuming that the channel state does not change during the transmission of a link layer frame, we define the channel state during transmission of frame $t$ as $S_{t}$, where $S_{t} \in\{1,2\}$. Value 1 and 2 correspond to the channel state "good" and "bad", respectively, where "good" channel state means the frames transmitted in such state are correctly received by the receiver, and "bad" channel means the frames transmitted in such state are not correctly received. Defining the transition probability $M_{i j}=$ Prob. $\left\{S_{t}=j \mid S_{t-1}=i\right\}$, the transition probability matrix of the Markov chain is given by:

$$
M=\left(\begin{array}{cc}
p & 1-p \\
1-q & q
\end{array}\right)
$$

For a Rayleigh fading channel, given the fading margin $F$, the Doppler frequency $f_{d}$ and the symbol (A symbol is an RLP frame here) length $T$, the transition matrix $M$ of the Markov chain, as well as the average frame error rate (FER) $P_{E}$ can be calculated. Because of limited space, we omitted the details of how to obtain $M$ and $P_{E}$ from $F$ and $f_{d} T$, which can be found in 8] [10]. Note that in the above model, the Frame Error Rate (FER) is solely decided by $F$, the fading margin, while the fading rate of the wireless channel depends on the product of $f_{d}$ and $T$. Large $f_{d} T$ means fast fading rate and short error burst, while small value of $f_{d} T$ implies slow fading rate and long error burst. When $f_{d} T=1$, the model reduces to i.i.d channel model. Note that $f_{d}=f\left(\frac{v}{c}\right)$, where $f$ is the carrier 
frequency, $v$ is the relative speed of mobile station to base station, and $c$ is the speed of light. For a particular channel, $f$ can be taken as a constant; when the link layer frame length $T$ is also fixed, the product $f_{d} T$ is solely dependent on $v$, the speed of the mobile station with respect to the base station.

\subsection{RLP Retransmission Efficiency}

A typical link layer ARQ used in wireless networks is Radio Link Protocol (RLP). The RLP is introduced into CDMA (IS95 and IS2000) systems in order to solve the TCP performance problem in wireless environment. Instead of letting TCP take care of the lost packets, the RLP tries to recover the lost link layer frames to avoid the unnecessary initiation of congestion control mechanism in upper TCP. The RLP is a negative acknowledgement (NACK) based Selective Repeat (SR) ARQ protocol. When a frame is detected to be in error or lost, copies of the lost frame are retransmitted. There are several optional retransmission schemes [8], including $(1,1,1,1,1,1)$ and $(1,2,3)$. Consider scheme $(1,2,3)$ for example: when RLP at the receiver side finds out that a frame is in error (or missing), it sends back two NACKs requesting for retransmission of this frame. A timer is set for this missing frame. The sender retransmits two copies of this frame. When the timer expires (times out) for the first time and this frame is still not received, three NACKs are sent back, the timer is reset, and each NACK received at the sender side triggers a retransmission of the missing frame so that three copies of the missing frame are transmitted. If the timer expires again, the RLP stops retransmission of this frame and sends whatever it has to the upper layer. For more details on RLP, refer to [14]16].

In this section, we study the retransmission efficiency of different RLP retransmission schemes both analytically and with simulation results. Note that an RLP frame can experience more than one transmission attempt before it is successfully received. For example, for scheme $(1,2,3)$, a frame can be successfully received in any of the six RLP transmission attempts. We say frames arriving at different RLP transmission attempts in different "phases". Formally, an RLP frame is in phase $i$ if it is received correctly for the first time at the $i$ th RLP transmission.

The probability of a frame in phase $i$ is given by:

$$
P_{h}(i)=\left\{\begin{array}{llr}
1-P_{E} & \text { if } & i=1 \\
S_{i}\left(M^{d(i)}\right)_{21} 1 & \text { if } 2 \leq i \leq \mid \text { scheme } \mid \\
S_{i} & \text { if } i=\mid \text { scheme } \mid+1
\end{array}\right.
$$

where $M^{d(i)}$ is matrix $M$ to the $d(i)$ th power, and $M_{i j}$ is the element at $i$ th row, $j$ th column of matrix $M$. |scheme| is the total number of RLP transmissions a frame can experience, which is dependent on particular RLP retransmission scheme. For example, for scheme $(1,2,3)$, $\mid$ scheme $\mid=1+2+3=6$. When $i=\mid$ scheme $\mid+1, P_{h}(i)$ means the frame is finally aborted by RLP since it has been transmitted for the maximum number of times that is allowed. $S_{i}$ is the probability that all previous transmission attempts fail, which is recursively given by: 
Table 1. $d(i)$ for RLP scheme $(1,2,3)$ and $(1,1,1,1,1,1)$, assuming RTT of 4 frames and RLP timeout value of 13 frames

\begin{tabular}{|c|c|c|}
\hline$i$ & $d(i)$ for $(1,2,3)$ & $d(i)$ for $(1,1,1,1,1,1)$ \\
\hline 1 & 0 & 0 \\
2 & 9 & 9 \\
3 & 10 & 23 \\
4 & 25 & 37 \\
5 & 26 & 51 \\
6 & 27 & 65 \\
7 & 35 & 73 \\
\hline
\end{tabular}

$$
S_{i}=\left\{\begin{array}{lrr}
0 & \text { if } & i=1 \\
P_{E} & \text { if } & i=2 \\
S_{i-1}\left(M^{d(i-1)}\right)_{22} & \text { if } 3 \leq i \leq \mid \text { scheme } \mid+1
\end{array}\right.
$$

For a frame in phase $i$, for $1 \leq i \leq \mid$ scheme $\mid+1, d(i)$ is the time that has passed (in unit of RLP frames) from the first reception of a frame to the time the frame is first correctly received or finally aborted by RLP. Note that different RLP retransmission schemes have different values of $d(i)$. Table 1 gives the values of $d(i)$ for scheme $(1,2,3)$ and $(1,1,1,1,1,1)$. Assuming that $d_{t}$ is the propagation delay from RLP sender to RLP receiver, $X(i)=d(i)+d_{t}$ is the delay seen by a frame that is in phase $i$. Thus the average delay of an RLP frame is:

$$
E(X)=\sum_{i=1}^{\mid \text {scheme }+1 \mid} \operatorname{Ph}(i) X(i)
$$

In order to study how different RLP schemes perform over correlated fading channels, and also to study the performance of different RLP retransmission schemes in terms of their efficiency in supporting TCP transmission, some analytic metric is needed. Because of the complexity in developing pure analytical model for TCP throughput with RLP retransmissions and correlated fading channel conditions, we use an indirect analytical metric, which is simple but provide enough insight into RLP's ability to support TCP in correlated fading channel.

Here we discuss the efficiency of two typical RLP retransmission schemes: $(1,2,3)$ and $(1,1,1,1,1,1)$. To develop a metric to evaluate the RLP performance of supporting TCP, both the probability of successful recovery and the time it takes for RLP to recover have to be taken into account. We first define $P_{r}$ to be the probability that, given the original transmission is in error, a frame is recovered during RLP retransmissions successfully. The complement probability $\overline{P_{r}}$ is given by:

$$
\overline{P_{r}}=\prod_{i=2}^{\mid \text {scheme } \mid-1}\left(M^{d(i)}\right)_{22}
$$


Note that the average delay $E(X)$ decides the speed of RLP recovery, while recovery rate $P_{r}$ decides the RLP's recovery capability.

Both of $E(X)$ and $P_{r}$ are important to the performance of RLP in supporting TCP connections. Hence, we take into consideration both of these two factors for defining the RLP retransmission efficiency $E F_{r}$. On the other hand, these two factors are not of the same magnitude, namely $0<P_{r}<1$, while $E(X) \geq 4$ assuming $d_{t} \geq 8$. It is not appropriate to use their direct ratio as the efficiency definition, because it magnifies the effect of $E(X)$ and ignoring the significance of $P_{r}$. we define $E F_{r}$ as

$$
E F_{r}=\frac{P_{r}}{\eta+E(X)^{\prime}}
$$

where $\eta$ is a tunable factor so that the relative significance of $P_{r}$ and $E(X)$ can be tuned. According to the simulations, we found that $\eta=10$ is appropriate for our purpose of evaluating the RLP scheme in supporting TCP. $E(X)^{\prime}$ is the normalized delay based on the maximum delay under consideration.

\subsection{Analytical and Simulation Results}

Fig. 2 shows the analytical results of $E F_{r}$ of two schemes under different channel conditions. First, it is observed that in fast fading channels $\left(f_{d} T=1\right.$, or i.i.d. channel), the RLP retransmission efficiency is better than in slow fading channels $\left(f_{d} T=0.01\right)$. This implies that slow fading channels, with high correlations of channel states between frames, are very unfriendly to SR-ARQs like RLP. Second, we observed a very interesting characteristic of RLP: when the degree of correlation is high (slow fading), scheme $(1,1,1,1,1,1)$ has better retransmission efficiency. While in low correlated channels (fast fading), scheme $(1,2,3)$ has better retransmission efficiency. To understand the reason behind this phenomenon, we look into the channel characteristics implied by slow and fast fading. Slow fading, i.e., large $f_{d} T$, means longer error burst length. In other words, if a frame is in error, there is a high possibility that the frame following the error frame is in error too (note that this probability is $M_{22}$ in the channel model). As a result, in scheme $(1,2,3)$, retransmitting 2 or 3 copies of the same frame in consecutive frames does not help much to improve the recovery probability. It has almost the same efficiency as the scheme $(1,1,1)$ in this case. Scheme $(1,1,1,1,1,1)$ is better because it just retransmits one copy of the lost frame at a time, thus significantly reducing the correlation between two retransmitted copies and having better recovery probability, in turn higher retransmission efficiency. When the degree of correlation is low, scheme $(1,2,3)$ has better efficiency. Because of the low correlation, recovery probability of scheme $(1,2,3)$ and $(1,1,1,1,1,1)$ are almost the same. But scheme $(1,2,3)$ has shorter average delay, which means it causes less delay in delivering the frames to upper layer, which makes the scheme $(1,2,3)$ perform better in this case.

We also study the RLP retransmission efficiency through simulations, and look at the RLP performance from another angle, TCP throughput, which is directly dependent on the RLP retransmission efficiency. The simulation is based 


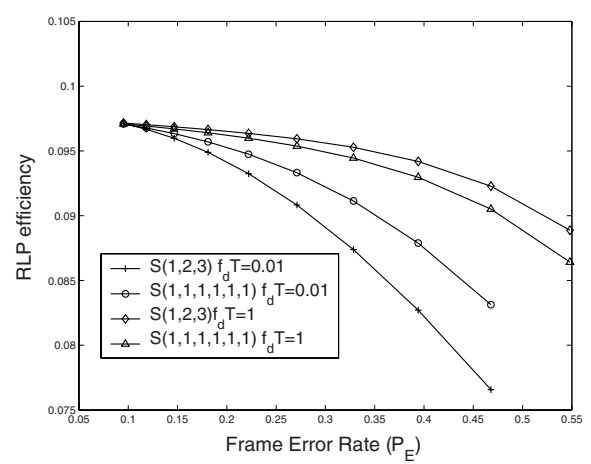

Fig. 2. RLP Retransmission Efficiency $\left(E F_{r}\right)$ vs. $P_{E}$

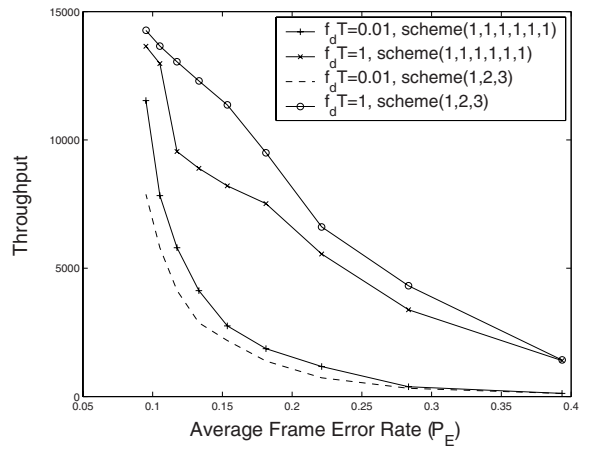

Fig. 3. TCP throughput in correlated channels vs. $P_{E}$

on Network Simulator 2 (NS2) [20]. Since NS2 does not provide RLP implementations, we developed RLP based on [14]. Other simulation parameters are: the RLP frame length is $5 \mathrm{~ms}$, i.e., an RLP frame is sent every $5 \mathrm{~ms}$. The wireless link bandwidth is assumed $2 \mathrm{Mbps}$, which is the common rate that 3rd generation wireless networks (such as IS2000 and UMTS) can provide. FTP is used as the application, which basically means the application always has enough data to transfer. TCP is used as the transport layer protocol with SACK option.

Fig. 3 shows the TCP throughput from simulation experiments. The TCP throughput shows the same characteristics as discussed above: RLP's overall performance is better in fast fading channels than in slow fading channels. In particular, in slow fading channels, scheme $(1,1,1,1,1,1)$ performs better, while in fast fading channels, scheme $(1,2,3)$ performance better. More importantly, the simulation results indicate that $E F_{r}$, the RLP retransmission efficiency, is a reasonable analytic metric for comparing the performances of different RLP schemes under correlated fading channels, especially when the supported transport layer protocol is TCP.

\section{An Adaptive RLP}

As we have pointed out, a single transmission scheme in RLP does not always yield the best performance. Sometimes, a mobile station may operate in highly correlated fading channels, while other times, it may operate in low correlated fading channels. This gives rise to the question of how we can achieve the best possible performance both in slow fading and fast fading channels with the RLP retransmission schemes that is most appropriate for the channel. The answer is to adaptively change the retransmission scheme based on the measured fading rate of the channel. Here we propose an adaptive RLP that embodies such strategy, which is called Adaptive Radio Link Protocol (ARLP). It is based on the RLP specifications of IS2000 systems 14.

Fig. 4 shows the basic functional units of the proposed ARLP. Because of the asymmetry of traffic load in uplink and downlink, most of bulk data transfer 
BSC/BS

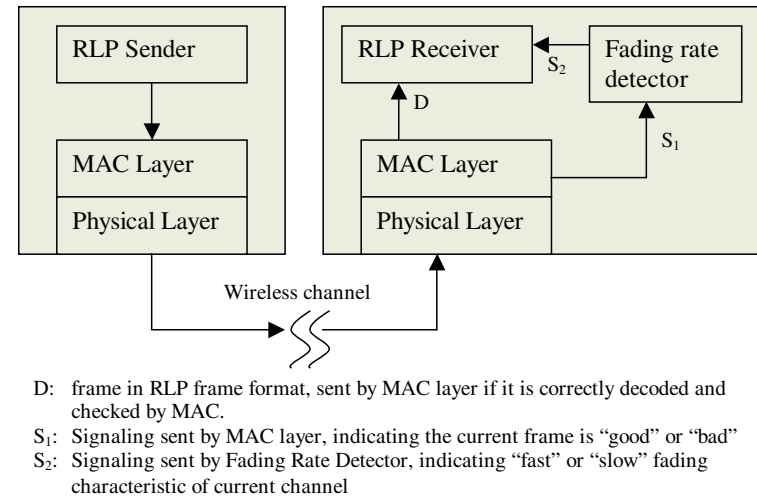

Fig. 4. Functional Units of ARLP

happens in the downlink direction, thus making the performance of down link more important in wireless networks for data. We will explain the application of the proposed ARLP mainly in downlink direction. Uplink can be treated similarly. If system complexity is a concern, the standard RLP can be used in uplink because the uplink traffic load is expected to be much lighter than downlink. The the performance improvement of utilizing ARLP in uplink may not be significant.

To detect the fading rate and its changes, some additional signaling is needed between the MAC layer and link layer. The MAC layer continuously decodes the frames received over the air interface from the base station. When a frame is decoded, no matter whether the frame is intended for this particular mobile station or not, the MAC sends a simple binary signal to the fading rate detector in link layer notifying the result of decoding: good frame or bad frame. This is different from most of the existing infrastructures where the MAC simply discards the frame if the decoding result indicates an error frame or a frame not for it. In such an infrastructure, the link layer is not aware of the discarded frames. As a result, most link layer adaptive protocols use ACK or NACK coming from receiver as the only source for channel estimate. However, for RLP, NACK is only sent when the RLP receiver finds gap(s) in the receiver buffer. This causes inefficient channel estimate and large delay in detecting change in channel characteristics, as we can easily see that a NACK received by sender indicates the channel condition almost a round trip time ago (or even longer because of bursty errors). Another reason justifying this inter-layer signaling is that we propose to do the channel estimate on the receiver side. The signaling between MAC layer and link layer in receiver significantly facilitates channel estimate. As proved in many earlier works, though the additional inter-layer interaction violates the principle of limiting the coupling of different layers of protocols as much as possible, sometimes it is necessary for wireless networks where efficiently utilizing the limited resources (e.g. bandwidth) is so important that it is worth to make a compromise. 


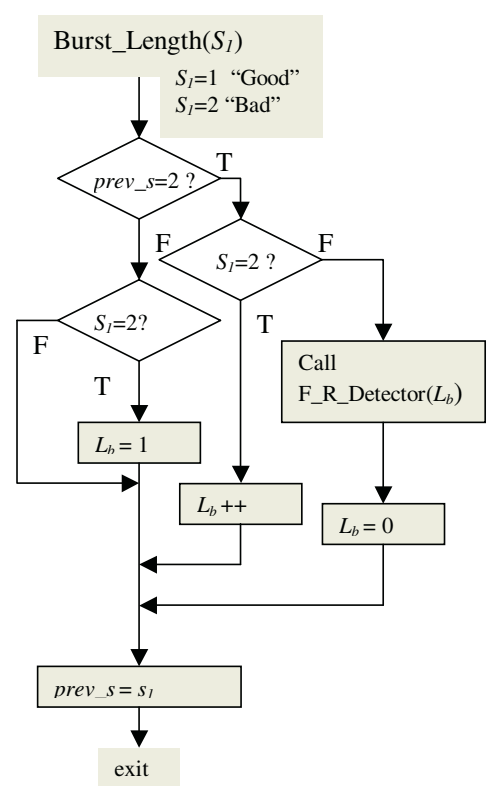

Fig. 5. Burst Length Sampling

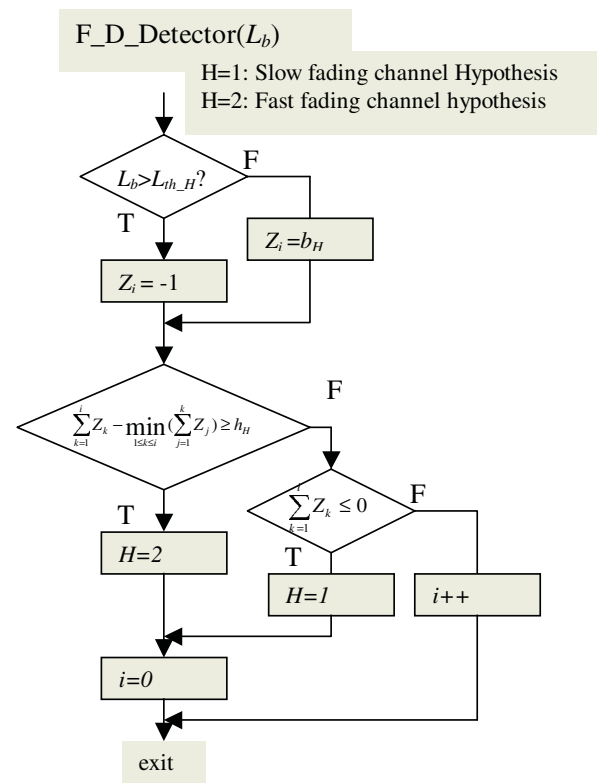

Fig. 6. Fading Rate Sequential Test

The purpose of fading rate detection is to estimate the channel fading rate, which is decided by the product of $f_{d}$ and $T$ in the correlated fading model. However, $f_{d} T$ is not a directly measurable parameter for a wireless receiver. Therefore, we have to resort to a measurable parameter that has close relationship with $f_{d} T$. The average length of frame error bursts $L_{b}=\frac{1}{1-q}$, is a monotonically decreasing function of $f_{d} T$, when the average frame error rate $P_{E}$ is fixed. Remember $q$ is the conditional probability Prob. $\left\{S_{t}=\right.$ "bad" $\mid S_{t-1}=$ "bad" $\}$. Since $L_{b}$ is a measurable parameter in receiver, we use it as the main source of channel fading rate estimate.

There are several test techniques to estimate characteristics of a random variable generated by a stochastic process. The problem of detecting the average burst length can be directly transformed into such a problem: given a sequence of frame error burst lengths, how can we tell if this is a fast fading channel or a slow fading channel? We choose the sequential test technique to do this detection because of its advantages over other adaptive detection techniques [5]. Unlike other test techniques, the sequential test does not have a fixed sample size. Also, it has short response time. Our algorithm consists of two parts: burst length calculation and fading rate detection. Fig. 5 and Fig. 6 respectively show the pseudocodes for these two functions: Burst_Length $\left(S_{1}\right)$ and F_D_Detector $\left(L_{b}\right)$. When the $(i-1)$ th frame is good, and the current $i$ th frame is bad, a new burst of error frames begins. The burst length counter $L_{b}$ keeps counting the number of continuous error frames until a good frame is detected. The length of this burst is then fed into the fading rate detector as a sample.

The fading rate detector is basically a sequential testing with two hypothesis: "fast fading channel" and "slow fading channel", which corresponds to short burst length and long burst length. Each burst length sample is scored based on 
its length and current hypothesis of the fading rate. If the current hypothesis is "slow fading channel", the score for each sample is given by:

$$
Z_{i}=\left\{\begin{array}{l}
-1 \text { if } L_{b}>L_{t h 1} \\
+b \text { if } L_{b} \leq L_{t h 1}
\end{array}\right.
$$

After the score of the current sample is calculated, if following condition is satisfied, the current hypothesis of slow fading channel is rejected:

$$
\sum_{k=1}^{i} Z_{k}-\min _{1 \leq k \leq i}\left(\sum_{j=1}^{k} Z_{j}\right) \geq h_{1}
$$

where $h_{1}$ is the threshold for rejecting the current hypothesis. The current hypothesis of slow fading channel is accepted when

$$
\sum_{k=1}^{i} Z_{k} \leq 0
$$

The sequential test restarts whenever the hypothesis is rejected or accepted. If the current hypothesis is rejected, the hypothesis is changed to the opposite one, which in our case is "fast fading channel", otherwise, current hypothesis is kept. The sequential test with "fast fading channel" hypothesis is done similarly to the test for "slow fading channel" hypothesis we have describe above. The current hypothesis, an indicator of current fading rate, is made a binary variable accessible to RLP receiver as the output of the fading rate detector. According to the value of this variable, the RLP receiver decides which retransmission scheme should be used for currently not correctly received frames. If the hypothesis is slow fading channel, scheme $(1,1,1,1,1,1)$ is used; otherwise, scheme $(1,2,3)$ is used. Note that the difference between these retransmission schemes is when to send NACKs and how many NACKs to send, which are actually decided by the RLP receiver. This is the reason why the channel fading rate estimate is done on the receiver side.

We set the default hypothesis as "fast fading channels" when the link is established. One may note that if the overall frame error rate $\left(P_{E}\right)$ is low, even in slow fading channels, the error frame burst length may not be long. Thus our fading rate estimate, solely based on average burst length could be wrong. However, this false estimate is not going to hurt the performance of the adaptive proposal, ARLP. Low $P_{E}$ indicates a fairly good channel condition. As we can see from the analysis and simulation results, in this case, the TCP throughput is indifferent to the retransmission scheme, because retransmission is rarely used.

\section{Performance}

\subsection{Response Time}

The time interval between a change in channel fading rate to the time the ARLP detects that change is defined as the response time. Fig. [7]shows the simulation 
results of average respond time to a typical channel fading rate change (from $f_{d} T=1$ to $\left.f_{d} T=0.01\right)$. We have observed that response time varies between 5 to 30 seconds. Also, the higher the $P_{E}$ is, the shorter the response time is. This response time is reasonable if we take into account that the sample here is the burst length, which is available only when a burst finishes. The interval between bursts can range from less than one second to several seconds long and certain number of samples are need to make an accurate estimate. This is the reason why a channel estimate mechanism can detect the change of BER in less than a second, while detecting fading rate change needs several seconds. On the other hand, this response time is fast enough for the purpose of fading rate detection. Though the signal strength may vary fast, the fading rate is unlikely to vary very fast. The variation of SIR (Signal to Interference Ratio) is caused by the mobility, while the variation of fading rate is caused by the variation of the speed of mobile stations. Note that fading rate is decided by $f_{d} T$, which is proportional to the speed of the mobile station. It is reasonable to assume that most of the mobile terminal users are not likely to vary their speed in a large range in a short period. Instead, most users tend to maintain their speed for a relatively long time (for at least several seconds). If they change the speed (e.g. from walking to riding a car), they are also likely to maintain that changed speed for a relatively long time. The response time of several seconds is fast enough to catch the change and respond with the appropriate retransmission schemes.

\subsection{TCP Performance Improvement}

We study the performance improvement of system with ARLP over systems with standard RLP through simulation experiments. We use the same simulation environment as described in Section 2.2

We first consider the scenario of one mobile user: a mobile user first walks for 80 seconds with a speed of $5 \mathrm{~km} /$ hour (about 3.10 miles/hour), which corresponds to $f_{d} T=0.02$, then the user gets on his car and moves for another 80 seconds with a speed of $96 \mathrm{~km} /$ hour (about 60miles/hour), which corresponds to $f_{d} T=0.4$. This scenario represent a typical fading rate change, thus is a good test for the proposed ARLP. The bulk data transmission happens during the whole process. Three systems are considered: a system of the proposed ARLP, a system of the standard RLP with scheme $(1,2,3)$ and a system of standard RLP with scheme $(1,1,1,1,1,1)$. Fig. 8 compares the TCP throughput of these three systems. We define the performance improvement as

$$
\delta=\frac{T_{A R L P}-\max \left(T_{123}, T_{111111}\right)}{\max \left(T_{123}, T_{111111}\right)}
$$

where $T_{A R L P}, T_{123}, T_{111111}$ are throughput of ARLP, standard RLP with scheme $(1,2,3)$ and standard RLP with scheme $(1,1,1,1,1,1)$ respectively. Fig. 9 (a) shows that the performance improvement can be as much as $10 \%$ for the simulated scenario, which is a significant improvement considering that it is achieved at a fairly high frame error rate of 0.12 .

In the second scenario, we consider the total TCP throughput improvement of the system. We assume a system with 100 users in full rate transmission. 20\% 


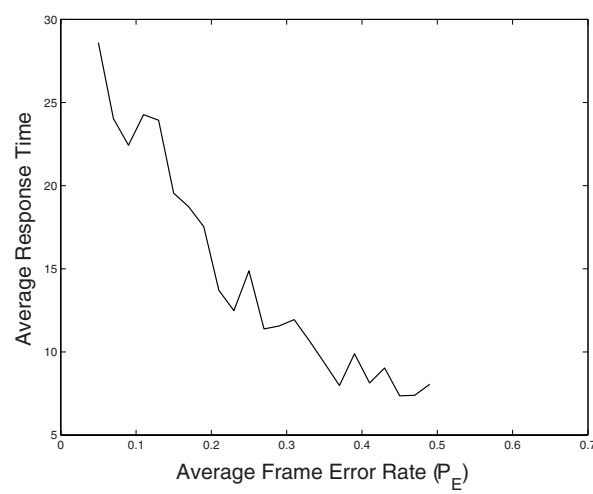

Fig. 7. Response Time vs. $P_{E}$

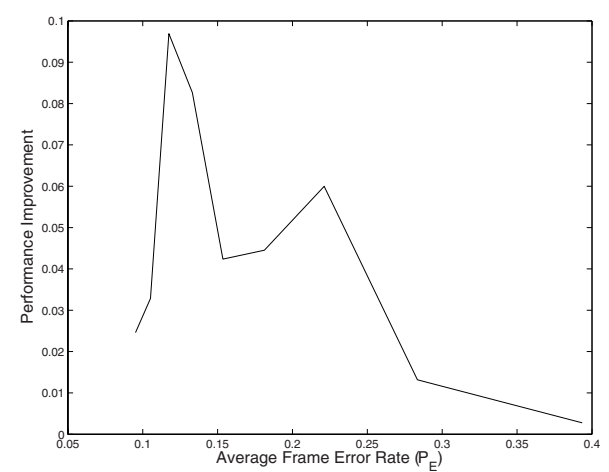

(a) Single User

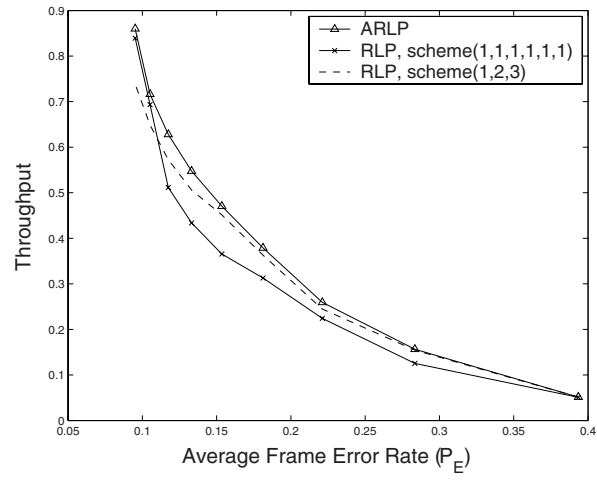

Fig. 8. Performance Comparison

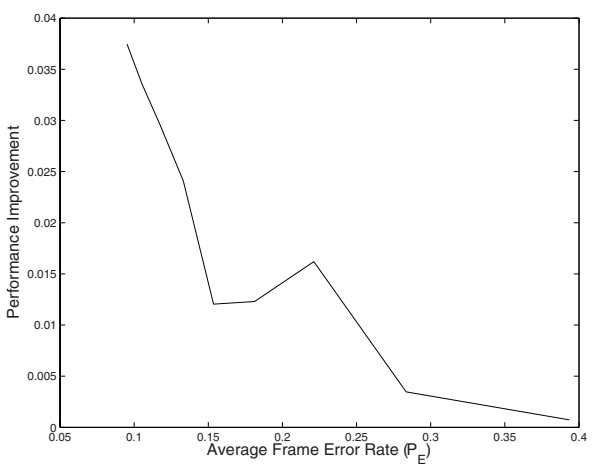

(b) System

Fig. 9. ARLP Performance Improvement

of the users are pedestrians with a speed of $5 \mathrm{~km} /$ hour, while $80 \%$ of the users are in vehicles with a speed of $96 \mathrm{~km} /$ hour. The same three systems as in single user scenario are considered. Throughput improvement has the same form of definition as (10), with $T_{A R L P}, T_{123}, T_{111111}$ representing the throughput of all users of each system instead of the throughput of a single user. Fig. 9 (b) shows the performance improvement $\delta$, with respect to average frame error rate $P_{E}$. It shows that the throughput of the system can also be improved up to $3.8 \%$ with ARLP.

It is observed that the most significant improvement always happens in moderate average frame error rate. This can be explained by noting that on one hand, the proposed ARLP works by optimizing retransmission schemes, which can only be effective when there are considerable errors, one the other hand, when the error rate is too high $\left(P_{E}>0.5\right)$, link layer recovery becomes inefficient and not much improvement can be expected. 


\section{Conclusion}

In this paper, we study the performance of different RLP retransmission schemes, especially scheme $(1,2,3)$ and scheme $(1,1,1,1,1,1)$, over correlated fading channels. We proposed a mathematically tangible metric: retransmission efficiency to evaluate the performance difference. We show that a single retransmission scheme does not result in best performance when the fading rate of correlated fading channels changes. To solve this problem, we propose a new adaptive RLP protocol ARLP, which uses sequential test technique to estimate the channel correlation, and adjusts its retransmission scheme according to the detected fading rate. Simulation results show that our ARLP can improve the TCP throughput performance when the user changes his/her moving speed, which results in changes in fading rate.

\section{References}

1. A.V Bakre and B.R. Badrinath, "I-TCP: indirect TCP for mobile hosts," International Conference on Distributed Computing Systems, 1995 pp. 136-143.

2. A.V Bakre and B.R Badrinath, "Implementation and performance evaluation of Indirect TCP," IEEE Trans. on Computers, Vol. 46 Issue 3, 1997 pp. 260-278.

3. S. Choi and K.G. Shin, "A class of adaptive hybrid ARQ schemes for wireless links," IEEE Trans. on Vehicular Technology, Vol. 50 Issue: 3, 2001 pp. 777-790.

4. H. Minn, M. Zeng and V.K. Bhargava, "On ARQ scheme with adaptive error control," IEEE Trans. on Vehicular Technology, Vol. 50 Issue: 6, 2001 pp. 14261436 .

5. M. Rice and S.B. Wicker, "A sequential scheme for adaptive error control over slowly varying channels," IEEE Trans. on Communications, Volume: 42 Issue: 2, 1994, pp. 1533-1543.

6. R. van Nobelen, M. Seshadri, J. Whitehead and S. Timiri, "An adaptive radio link protocol with enhanced data rates for GSM evolution," IEEE Personal Communications, Volume: 6 Issue 1, 1999 pp. 54-64.

7. P. Lettieri and M. B. Srivastava, "Adaptive frame length control for improving wireless link throughput, range, and energy efficiency," INFOCOM 98, Volume: 2, 1998 pp. $564-571$.

8. A. Chockalingam and G. Bao, "Performance of TCP/RLP protocol stack on correlated fading DS-CDMA wireless links," IEEE Transactions on Vehicular Technology, Volume: 49, 2000 pp. 28-33.

9. H.S.Hang, "On verifying the first-order Markovian assumption for a Rayleigh fading channel model," Proc IEEE ICUPC, 1994, pp. 160-164.

10. M. Zorzi, R.R. Rao and L.B. Milstein, "On the accuracy of a first-order Markov model for data block transmission on fading channels," IEEE ICUPC'95, pp. 211215.

11. P. Sinha, N. Venkitaraman, R. Sivakumar and V. Bharghavan, "WTCP: A Reliable Transport Protocol for Wireless Wide-Area Networks," ACM Mobicom, Seattle. WA. August 1999.

12. 3G Partnership Project, Release 99

13. M. Rice and S.B. Wicker, "Adaptive error control for slowly varying channels," IEEE Transactions on Communications, February-April 1994 pp. 917-926. 
14. TIA/EIA/IS-707-A-2.10, "Data Service Options for Spread Spectrum Systems: Radio Link Protocol Type 3," January 2000.

15. S. Souissi and S.B. Wicher, "A diversity combining DS/CDMA system with convolutional encoding and Viterbi Decoding," IEEE Trans. on Vehicular Technology, Vol44, NO.2, May 1995 pp. 304-312.

16. J. M. Harris and M. Airy, "Analytical model for radio link protocol for IS-95 CDMA systems," Vehicular Technology Conference, 2000. Vol. 3 pp. 2434-2438.

17. M. Abramowitz and I. A. Stegun, "Bessel Functions J and Y." §9.1 Handbook of Mathematical Functions with Formulas, Graphs, and Mathematical Tables, 9th printing. New York: Dover, pp. 358-364, 1972.

18. M. Schwartz, W. R. Bennett and S. Stein, Communication Systems and Techniques, New York: McGraw-Hill, 1966

19. H. Lin and S. K. Das, "TCP performance analysis of CDMA systems with RLP and MAC layer retransmissions," 10th IEEE International Symposium on Modeling, Analysis and Simulation of Computer and Telecommunications Systems (MASCOTS 2002), pp. 313-320.

20. The Network Simulator - ns-2, http://www.isi.edu/nsnam/ns/ 